\title{
CD40L-Fc Fusion Protein MEDI5083
}

National Cancer Institute

\section{Source}

National Cancer Institute. CD40L-FC Fusion Protein MEDI5083. NCI Thesaurus. Code C148416.

A fusion protein composed of CD40 lig and (CD40L; CD154; TRAP; TNFSF5) fused to a modified immunoglobulin (Ig) Fc fragment, with potential immunostimulating activity. Upon administration of the CD40L-Fc fusion protein MEDI5083, the CD40L moiety specifically targets, binds to, and activates CD40, a cell surface receptor that belongs to the tumor necrosis factor (TNF) receptor family and is expressed on various immune cells, such as B-lymphocytes, monocytes, and dendritic cells (DCs). Activation of CD40 induces proliferation and activation of B-lymphocytes, shifts the induction of suppressive macrophages towards immunostimulatory macrophages, activates monocyte-derived DCs (moDCs), and leads to the secretion of inflammatory cytokines, which activates the immune system to induce the proliferation and activation of cytotoxic T-lymphocytes (CT Ls) against tumor cells. Altogether, this may cause tumor cell lysis. 